\title{
ARBITRAGE IN A SIMPLE MODEL WITH GENERAL TRANSACTION COSTS
}

Abstract. We study a version of no arbitrage condition in a simple model with general transaction costs. Our condition is equivalent to the existence of an equivalent martingale measure.

1. Introduction. In classical models of stock markets a non-arbitrage condition implies the existence of a martingale measure (see $[\mathrm{HK}]$ ), which is crucial in pricing theory. If transaction costs are assumed, any "proper", reasonably general no-arbitrage condition is essentially weaker than the existence of a martingale measure. Some classical results in this direction can be found in: [JK1], [JK2], [KRS1], [KRS2], [PT].

Recently J. Piasecka introduced a condition which proves to be equivalent to the existence of a martingale measure in the case of independent increments of prices (see [P1], [P2]). Roughly speaking, every known approach defines arbitrage as an opportunity of obtaining a strictly positive final capital without incurring any risk. The point is which initial market positions of arbitrage strategy are accepted. In particular Piasecka allows any strategy starting from a position from which only zero ("have nothing") position can be obtained without stock prices being changed.

In this paper we generalize the result of Piasecka. We discuss a specific model of one-stock market in a finite time with arbitrary transaction costs and obtain the equivalence of a Piasecka-type no-arbitrage condition to the existence of an equivalent martingale measure which is presented in an explicit form. Similar calculations can be found in [CP]. The main results of our paper are given in Theorems 2.1 and 3.1.

2000 Mathematics Subject Classification: 91B28, 91B70.

Key words and phrases: arbitrage, transaction costs, martingale measures.

The author is grateful to Prof. Adam Paszkiewicz for his helpful advice and comments. Research supported by KBN grant "Metody matematyczne w analizie rynków i instrumentów finansowych w Polsce" 2001-04. 
2. The model. Consider a probability space $(\Omega, \mathcal{F}, P)$, a filtration $\left(\mathcal{F}_{t}\right)_{t=0, \ldots, T}$ and a nonnegative, adapted price process of one stock $\left(S_{t}\right)_{t=0, \ldots, T}$, where $S_{t} \in L_{t}^{\infty} \equiv L^{\infty}\left(\Omega, \mathcal{F}_{t}, \mathbb{R}\right)$ for all $t$. Let $\xi_{t}=\frac{S_{t}-S_{t-1}}{S_{t-1}} \in L_{t}^{1}$. Assume that a function $\eta: \mathbb{R} \rightarrow \mathbb{R}$ describes transaction costs as follows:

- if $y>0$ then $\eta(y)$ is the amount of money obtained from selling $y$ worth of stock,

- if $y<0$ then $-\eta(y)$ is the amount of money necessary to buy $-y$ worth of stock.

Furthermore it is reasonable to assume that the following conditions are satisfied:

$$
\begin{aligned}
& \eta(0)=0, \\
& \forall_{x, y \in \mathbb{R}} \quad \eta(x)+\eta(y) \leq \eta(x+y) \quad \text { (superadditivity), } \\
& \exists_{\alpha>1} \forall_{x>0} \quad 0<\eta(x) \leq x \wedge-\eta(-x) \leq \alpha x .
\end{aligned}
$$

Our market position at every moment is defined by an ordered pair of real numbers $(x, y)$. The numbers $x, y$ denote respectively the amount of cash the investor possesses and the value of stocks in his portofolio. By strategy we will understand an adapted process $\left(m_{t}\right)_{t=0, \ldots, T-1}$, which is interpreted as the value of stocks the investor purchases at time $t$. Let us introduce the following notation:

$$
\begin{aligned}
& C_{0}=\left\{(x, y) \in \mathbb{R}^{2}: x+\eta(y) \geq 0\right\}, \\
& C_{b}=\left\{(x, y) \in \mathbb{R}^{2}: x+\eta(y)=0\right\}, \\
& C_{i}=\left\{(x, y) \in \mathbb{R}^{2}: x+\eta(y)>0\right\} .
\end{aligned}
$$

Definition 1. We shall say that there exists an arbitrage opportunity or an arbitrage at time $t \in\{1, \ldots, T\}$ if for some integrable $\mathcal{F}_{t-1}$-measurable random variables $X, Y$ such that

we have

$$
\langle X, Y\rangle \in C_{b} \quad P \text {-a.s., }
$$

$$
\left\langle X,\left(1+\xi_{t}\right) Y\right\rangle \in C_{0} \quad P \text {-a.s. } \quad \text { and } \quad P\left(\left\langle X,\left(1+\xi_{t}\right) Y\right\rangle \in C_{i}\right)>0 .
$$

DEFINITION 2. We shall say that there exists an arbitrage opportunity or an arbitrage in the interval $[0, T]$ if for some $\mathcal{F}_{0}$-measurable random variables $X_{0}, Y_{0}$ such that

$$
\left\langle X_{0}, Y_{0}\right\rangle \in C_{b} \quad P \text {-a.s. }
$$

and an adapted $\left(m_{t}\right)_{t \in\{0, \ldots, T-1\}}, m_{t} \in L_{t}^{1}$,

$$
P\left(\mathbb{H}_{T} \in C_{i}\right)>0 \text { and } \mathbb{H}_{T} \in C_{0} P \text {-a.s., }
$$

where $\mathbb{H}_{T}$ is the investor's market position at time $T$, i.e.

$$
\mathbb{H}_{T} \equiv\left\langle X_{0}-\sum_{t=0}^{T-1} \eta\left(-m_{t}\right), Y_{0} \cdot \prod_{i=1}^{T}\left(1+\xi_{i}\right)+\sum_{t=0}^{T-1}\left(\prod_{i=t}^{T}\left(1+\xi_{i}\right)\right) \cdot m_{t}\right\rangle .
$$


Our main result is the following.

THEOREM 2.1. If there is no arbitrage opportunity at any time $t \in$ $\{1, \ldots, T\}$ then:

(a) there is a measure $Q \sim P$ (equivalent to $P$ ) such that

$$
\forall_{t \leq N} \quad E_{Q}\left(S_{t}-S_{t-1} \mid \mathcal{F}_{t-1}\right)=0,
$$

(b) there is no arbitrage opportunity in the interval $[0, T]$.

It is natural to expect that (a) implies the lack of arbitrage at any time. We prove this as Theorem 3.1.

\section{Proof of the main results}

Proof of Theorem 2.1. First we prove (a). Assume the absence of arbitrage at every time $t$, i.e.

$\forall_{t \leq T} \sim \exists_{X, Y \in L^{1}\left(\Omega, \mathcal{F}_{t-1}, C_{b}\right)}\left[\left\langle X,\left(1+\xi_{t}\right) Y\right\rangle \in C_{0}\right.$ p.p. $\left.\wedge P\left(\left\langle X,\left(1+\xi_{t}\right) Y\right\rangle\right)>0\right]$.

It follows that

$$
\forall_{0<t \leq T} \forall_{A \in \mathcal{F}_{t-1}} \quad\left[P\left(A \cap\left\{\xi_{t}>0\right\}\right)>0 \Leftrightarrow P\left(A \cap\left\{\xi_{t}<0\right\}\right)>0\right] .
$$

Indeed, if there were a set $A$ such that for instance $P\left(A \cap\left\{\xi_{t} \geq 0\right\}\right)>0$ then for $\langle X, Y\rangle \equiv\left\langle-\eta\left(\mathbb{I}_{A}\right), \mathbb{I}_{A}\right\rangle$ one would have an arbitrage opportunity at time $t$.

Set $D_{n}=S_{n}-S_{n-1}$. The existence of the required measure will be proved inductively. We will show that for every $n<T$ there is a measure $P_{n}$ satisfying

$$
\forall_{0<t \leq n} \quad E_{P_{n}}\left(D_{t} \mid \mathcal{F}_{t-1}\right)=0 .
$$

Set $P_{0}=P$ and note that the foregoing holds true for $n=0$. Assume that for some $n<T$ there is such $P_{n} \sim P$.

We shall prove that

$$
\begin{aligned}
W= & E_{P_{n}}\left(D_{n+1}^{+} \mid \mathcal{F}_{n}\right) \cdot P_{n}\left(D_{n+1}<0 \mid \mathcal{F}_{n}\right) \\
& +E_{P_{n}}\left(D_{n+1}^{-} \mid \mathcal{F}_{n}\right) \cdot P_{n}\left(D_{n+1}>0 \mid \mathcal{F}_{n}\right)>0
\end{aligned}
$$

whenever $D_{n+1} \neq 0$. Let $A=\{W=0\} \in \mathcal{F}_{n}$. Assume that for example

$$
P\left(A \cap\left\{D_{n+1}>0\right\}\right)>0 .
$$

Then the conditional expectation $E_{P_{n}}\left(D_{n+1}^{+} \mid \mathcal{F}_{n}\right)$ is nonzero on $A$ with positive probability. Set

$$
A^{\prime}=A \cap\left\{E_{P_{n}}\left(D_{n+1}^{+} \mid \mathcal{F}_{n}\right)>0\right\} .
$$

It is not hard to notice that $P\left(A^{\prime} \cap\left\{D_{n+1}>0\right\}\right)>0$ and by (1) also $P\left(A^{\prime} \cap\left\{D_{n+1}<0\right\}\right)>0$. Thus $E_{P_{n}}\left(D_{n+1}^{+} \mid \mathcal{F}_{n}\right) \cdot P_{n}\left(D_{n+1}<0 \mid \mathcal{F}_{n}\right)>0$ with positive probability on $A^{\prime} \subset A$. That is a contradiction. 
Let us define a function $\varphi_{n}: \Omega \rightarrow \mathbb{R}$ as follows:

$$
\varphi_{n}= \begin{cases}X & \text { if } D_{n+1}>0 \\ 1 & \text { if } D_{n+1}=0 \\ Y & \text { if } D_{n+1}<0\end{cases}
$$

where the functions $X$ and $Y$ solve the following system:

$$
\left\{\begin{array}{l}
X \cdot E_{P_{n}}\left(D_{n+1}^{+} \mid \mathcal{F}_{n}\right)-Y \cdot E_{P_{n}}\left(D_{n+1}^{-} \mid \mathcal{F}_{n}\right)=0 \\
X \cdot P_{n}\left(D_{n+1}>0 \mid \mathcal{F}_{n}\right)+Y \cdot P_{n}\left(D_{n+1}<0 \mid \mathcal{F}_{n}\right)=P_{n}\left(D_{n+1} \neq 0 \mid \mathcal{F}_{n}\right),
\end{array}\right.
$$

and are unique almost surely on $\left\{D_{n+1} \neq 0\right\}$. Let the measure $P_{n+1}$ be defined by $d P_{n+1}=\varphi_{n} \cdot d P_{n}$. Since clearly $\varphi_{n}>0$, we conclude that $P_{n+1} \sim$ $P_{n} \sim P$.

Notice that $P_{n+1}=P_{n}$ on $\mathcal{F}_{n}$. If $A \in \mathcal{F}_{n}$ then

$$
\begin{aligned}
P_{n+1}(A) & =E_{P_{n+1}} \mathbb{I}_{A}=E_{P_{n}}\left(\varphi_{n} \cdot \mathbb{I}_{A}\right)=E_{P_{n}}\left(\mathbb{I}_{A} \cdot E_{P_{n}}\left(\varphi_{n} \mid \mathcal{F}_{n}\right)\right) \\
& =E_{P_{n}}\left(\mathbb{I}_{A} \cdot E_{P_{n}}\left(X \cdot \mathbb{I}_{\left\{D_{n+1}>0\right\}}+Y \cdot \mathbb{I}_{\left\{D_{n+1}<0\right\}}+\mathbb{I}_{\left\{D_{n+1}=0\right\}} \mid \mathcal{F}_{n}\right)\right) \\
& =E_{P_{n}}\left(\mathbb{I}_{A} \cdot\left(P_{n}\left(D_{n+1} \neq 0 \mid \mathcal{F}_{n}\right)+P_{n}\left(D_{n+1}=0 \mid \mathcal{F}_{n}\right)\right)\right) \\
& =E_{P_{n}}\left(\mathbb{I}_{A}\right)=P_{n}(A) .
\end{aligned}
$$

We now show that $P_{n+1}$ satisfies condition (2). If $k=n$, then

$$
\begin{aligned}
E_{P_{n+1}}\left(D_{k} \mid \mathcal{F}_{k}\right) & =E_{P_{n+1}}\left(D_{n} \mid \mathcal{F}_{n}\right)=E_{P_{n}}\left(\varphi_{n} \cdot D_{n} \mid \mathcal{F}_{n}\right) \\
& =E_{P_{n}}\left(X \cdot D_{n}^{+} \mid \mathcal{F}_{n}\right)+E_{P_{n}}\left(Y \cdot D_{n}^{-} \mid \mathcal{F}_{n}\right)+E_{P_{n}}\left(0 \mid \mathcal{F}_{0}\right) \\
& =X \cdot E_{P_{n}}\left(D_{n}^{+} \mid \mathcal{F}_{n}\right)+Y \cdot E_{P_{n}}\left(D_{n}^{-} \mid \mathcal{F}_{n}\right)=0 .
\end{aligned}
$$

In case $k<n$,

$$
E_{P_{n+1}}\left(D_{k+1} \mid \mathcal{F}_{k}\right)=E_{P_{n}}\left(D_{k+1} \mid \mathcal{F}_{k}\right)=0
$$

by inductive assumption. In this way, after $T$ steps, a measure $Q=P_{T}$ is obtained such that $Q \sim P$ as well as

$$
\forall_{t \leq N} \quad E_{Q}\left(S_{t}-S_{t-1} \mid \mathcal{F}_{t-1}\right)=0 .
$$

Now we prove (b). Let

$$
t_{0}=\min \left\{t \in \mathbb{N}: P\left(\mathbb{H}_{t} \in C_{0}\right)=1 \wedge P\left(\mathbb{H}_{t} \in C_{i}\right)>0\right\} .
$$

Then we have two possibilities:

- $P\left(\mathbb{H}_{t_{0}-1} \in C_{b}\right)=1$,

- $P\left(\mathbb{H}_{t_{0}-1} \notin C_{0}\right)>0$.

In the first case there is clearly an arbitrage opportunity at time $t_{0}$. In the second case consider the following sets:

$$
\begin{aligned}
& A_{+}=\left\{\mathbb{H}_{t_{0}-1} \notin C_{0}\right\} \cap\left\{Y_{t_{0}-1}+m_{t_{0}-1}>0\right\}, \\
& A_{-}=\left\{\mathbb{H}_{t_{0}-1} \notin C_{0}\right\} \cap\left\{Y_{t_{0}-1}+m_{t_{0}-1}<0\right\} .
\end{aligned}
$$


Then either of the two market positions $\left\langle-\eta\left(\mathbb{I}_{A_{+}}\right), \mathbb{I}_{A_{+}}\right\rangle$and $\left\langle-\eta\left(\mathbb{I}_{A_{-}}\right), \mathbb{I}_{A_{-}}\right\rangle$ leads to an arbitrage at time $t_{0}$.

THEOREM 3.1. If there exists a measure $Q \sim P$ such that

$$
\forall_{t \leq N} \quad E_{Q}\left(S_{t}-S_{t-1} \mid \mathcal{F}_{t-1}\right)=0,
$$

then there is no arbitrage in the interval $[0, T]$.

Proof. Assume that there exists a measure $Q$ as above. The process $D_{t}$ and hence also $\xi_{t}$ must satisfy condition (1). Indeed, if for example

$$
P\left(A \cap\left\{D_{t}>0\right\}\right)>0 \wedge P\left(A \cap\left\{D_{t}<0\right\}\right)=0
$$

for some $A \in \mathcal{F}_{t}$ then $D_{t} \geq 0$ on $A$ and $\int_{A} D_{t} d P>0$ as well as

$$
\int_{A} E_{Q}\left(D_{t} \mid \mathcal{F}_{t}\right) d Q=\int_{A} D_{t} d Q>0
$$

This contradicts $E_{Q}\left(D_{t} \mid \mathcal{F}_{t}\right)=0$.

Let $X_{0}, Y_{0} \in L_{0}^{1}$ be such that $X_{0}+\eta\left(Y_{0}\right)=0$ and let $\left(m_{t}\right)_{t<T}$ be a strategy. It will be shown inductively that for every $0 \leq t \leq T$ the following alternative is true:

$$
X_{t}+\eta\left(Y_{t}\right)=0 \vee P\left(X_{t}+\eta\left(Y_{t}\right)<0\right)>0 .
$$

Assume that the foregoing is true for some $t<T$.

If $X_{t}+\eta\left(Y_{t}\right)=0$ then one of the following is true:

(a) $P\left(Y_{t}+m_{t}<0\right)>0$,

(b) $P\left(Y_{t}+m_{t}>0\right)>0$,

(c) $Y_{t}+m_{t}=0$.

In case (a) by (1) either $\xi_{t}=0$ on $A=\left\{Y_{t}+m_{t}<0\right\}$ or $P\left(A \cap\left\{\xi_{t}>0\right\}\right)$ $>0$. In the former case we have

$$
\begin{aligned}
X_{t+1}+\eta\left(Y_{t+1}\right) & =X_{t}-\eta\left(-m_{t}\right)+\eta\left(\left(1+\xi_{t}\right) \cdot\left(Y_{t}+m_{t}\right)\right) \\
& =X_{t}-\eta\left(-m_{t}\right)+\eta\left(Y_{t}+m_{t}\right) \leq 0
\end{aligned}
$$

almost surely on $A$, and in the latter

$$
\begin{aligned}
X_{t+1}+\eta\left(Y_{t+1}\right) & =X_{t}-\eta\left(-m_{t}\right)+\eta\left(\left(1+\xi_{t}\right) \cdot\left(Y_{t}+m_{t}\right)\right) \\
& <X_{t}-\eta\left(-m_{t}\right)+\eta\left(Y_{t}+m_{t}\right) \leq 0
\end{aligned}
$$

with positive probability on $A$. Case (b) can be treated similarly, and (c) is obvious.

If $X_{t}+\eta\left(Y_{t}\right)<0$ the same considerations as in cases (a)-(c) lead to the conclusion that

$$
P\left(X_{t+1}+\eta\left(Y_{t+1}\right)<0\right)>0 .
$$

This completes the induction. 
4. Final remarks. Once we have Theorems 2.1 and 3.1, the following question arises almost immediately. Does an arbitrage opportunity at one particular time imply arbitrage in the whole interval? A simple counterexample yields a negative answer. It is sufficient to consider $\Omega$ such that $\# \Omega=2$ and $\xi_{1}=\langle 1,0.5\rangle, \xi_{2}=\langle 1,0\rangle, \eta(x)=3 x$. There is clearly an arbitrage at $t=1$ but no possibility of making a profit without risk when $\mathcal{F}_{0}=\{\emptyset, \Omega\}$.

In [P2] Piasecka studied some particular cases of transaction costs and obtained equivalence of the existence of arbitrage in a given step and the existence of arbitrage in the whole interval. The key assumption was that $\xi_{t}$ were i.i.d. and $P\left(\left\{\xi_{t}=0\right\}\right)=0$. The latter seems unnecessary and we think it could be simply omitted.

A different definition of arbitrage opportunity was introduced by Pham and Thouzi in $[\mathrm{PT}]$. They accepted only those starting positions in arbitrage strategy which could be "bought" from $(0,0)$. In that case there is no arbitrage possibility for some markets without a martingale measure.

\section{References}

[CP] E. Czkwianianc and A. Paszkiewicz, On martingale measures for stochastic processes with discrete time, Probab. Math. Statist. 19 (1999), 203-209.

[HK] J. M. Harrison and D. M. Kreps, Martingales and arbitrage in multiperiod securities markets, J. Econom. Theory 20 (1979), 381-408.

[JK1] E. Jouini and H. Kallal, Arbitrage in securities markets with short-sales constraints, Math. Finance 5 (1995), 197-232.

[JK2] - - - Martingales and arbitrage in securities markets with transaction costs, J. Econom. Theory 66 (1995), 178-197.

[KRS1] Yu. Kabanov, M. Rásonyi and Ch. Stricker, No-arbitrage criteria for financial markets with efficient friction, Finance Stoch. 6 (2002), 371-382.

[KRS2] - - - - On the closedness of sums of convex cones in $L^{0}$ and the robust no-arbitrage property, ibid. 7 (2003), 403-411.

[PT] H. Pham and N. Touzi, The fundamental theorem of asset pricing with cone constraints, J. Math. Econom. 31 (1999), 265-279.

[P1] J. Piasecka, Discrete time arbitrage under transaction costs, Appl. Math. (Warsaw) 27 (2000), 419-436.

[P2] - , Selected problems of markets with various transaction costs, $\mathrm{PhD}$ thesis, Maria Curie-Skłodowska Univ., Lublin, 2003 (in Polish).

Department of Mathematics

University of Łódź

Banacha 22

90-238 Łódź, Poland

E-mail: jakubo@math.uni.lodz.pl

Received on 13.7.2004; 